\title{
Effects of rigid or adaptive confinement on colloidal self-assembly. Fixed vs. fluctuating number of confined particles
}

\author{
J. Pȩkalski, ${ }^{1}$ N. G. Almarza, ${ }^{2}$ and A. Ciach ${ }^{1}$ \\ ${ }^{1}$ Institute of Physical Chemistry, Polish Academy of Sciences, 01-224 Warszawa, Poland \\ ${ }^{2}$ Instituto de Química Física Rocasolano, CSIC, Serrano 119, E-28006 Madrid, Spain
}

(Received 15 April 2015; accepted 15 May 2015; published online 28 May 2015)

\begin{abstract}
The effects of confinement on colloidal self-assembly in the case of fixed number of confined particles are studied in the one dimensional lattice model solved exactly in the grand canonical ensemble (GCE) in Pȩkalski et al. [J. Chem. Phys. 142, 014903 (2015)]. The model considers a pair interaction defined by a short-range attraction plus a longer-range repulsion. We consider thermodynamic states corresponding to self-assembly into clusters. Both fixed and adaptive boundaries are studied. For fixed boundaries, there are particular states in which, for equal average densities, the number of clusters in the GCE is larger than in the canonical ensemble. The dependence of pressure on density has a different form when the system size changes with fixed number of particles and when the number of particles changes with fixed size of the system. In the former case, the pressure has a nonmonotonic dependence on the system size. The anomalous increase of pressure for expanding system is accompanied by formation of a larger number of smaller clusters. In the case of elastic confining surfaces, we observe a bistability, i.e., two significantly different system sizes occur with almost the same probability. The mechanism of the bistability in the closed system is different to that of the case of permeable walls, where the two equilibrium system sizes correspond to a different number of particles. (C) 2015 AIP Publishing LLC. [http://dx.doi.org/10.1063/1.4921787]
\end{abstract}

\section{INTRODUCTION}

Confinement has a significant effect on fluids when the separation between the confining surfaces is comparable with the characteristic structural length of the confined fluid. ${ }^{1,2}$ In simple fluids, such pore thicknesses are of order of Ångströms. The effects of confinement depend on whether the boundaries are rigid or adaptive. In the latter case, mechanical equilibrium between the stress in the solid walls resulting from the swelling or shrinking of the pore and the solvation force ${ }^{1,2}$ induced on the walls by the confined fluid can lead to contraction or expansion of the pore. ${ }^{3}$ Similar dependence on the type of confinement is expected in the case of self-assembling systems, but on different length and energy scales. ${ }^{4-10}$ The packing effects of molecules are replaced in this case by the packing effects of micelles, clusters, or layers that are much larger and softer.

In this work, we focus on colloidal self-assembly in thermodynamic conditions corresponding to self-assembly into clusters or layers in the bulk. ${ }^{8,11-15}$ We study rigid and elastic boundaries, and consider separations of the confining walls comparable with a few structural units. The adaptive confinement in this case means confining surfaces that are soft and separated by hundreds of nanometers. Important examples of such a confinement exist in organelles or in lipid or polymeric vesicles. One can expect that the shape of the outer membrane and the ordering inside the vesicle or organelle influence each other in a way that depends on the elasticity of the membrane. We shall consider self-assembly in systems with fixed boundaries and with boundaries with different elasticities, from stiff to very soft.

Some of the membranes are permeable, while some other are not. Thus, a question arises if fluctuations of the number of confined particles have any effect on the properties of a selfassembling system confined by rigid or adaptive boundaries. This question motivates our present study. We ask how the effects of confinement on the self-assembling system depend on the contact with a reservoir of particles.

The above problems are very difficult for realistic models of self-assembling systems confined by elastic boundaries. In order to gain some first insight, however, one can consider simplified models. In this work, we consider the simple generic model of the system with competing short-range attraction and longer-range repulsion (SALR) that can be solved exactly. ${ }^{16}$ The model was solved exactly and systematically analyzed in the bulk ${ }^{16-18}$ and in confinement ${ }^{19}$ in the grand canonical ensemble (GCE). Recently, a procedure to extract full canonical information from grand-canonical results has been proposed in Ref. 20. In principle, it is possible to apply this technique to our exact GCE results. However, in the current problem, the presence of energetic terms and low temperature pose numerical difficulties in the mapping procedure between the results in both ensembles. For this reason, we decide to perform Monte Carlo (MC) simulations in the canonical ensemble (CE).

In the case of fixed boundaries, we address the question of how the fluctuations of the positions and sizes of the clusters (their dynamical assembly and dissociation) are coupled with 
the fluctuations of the number of particles in the system. In the case of inhomogeneous distribution of particles, it is not $a$ priori obvious that the largest fluctuations in the total number of particles lead to the largest differences between the density profiles in the canonical and grand canonical ensembles. We shall compare the density profiles and the pressure in the GCE and CE ensembles, with the average number of particles in the GCE equal to the number of particles $N_{0}$ in the CE. We shall pay particular attention to values of $N_{0}$ that are too small or too large for a given system size $L$ for formation of periodically distributed layers of particles that minimizes the system energy. Roughly speaking, in the SALR systems, the minimum energy is assumed when the individual clusters have the lowest energy (no intra-cluster repulsion) and are separated by the smallest distances corresponding to no inter-cluster repulsion. When the number of particles is too small or too large for a given $L$ for formation of the optimal bulk structure, some structural deformations must occur. Our purpose is to compare these deformations and their effect on the mechanical properties in the GCE and CE.

Adaptive boundaries were studied in Ref. 21 in the case of fixed number of discs surrounded by particles kept by laser tweezers. The system exhibited bistability; either hexagonal structure (and modified boundaries) or concentric rings of particles occurred. We observed a different bistability in Ref. 19, where we studied particles interacting with the SALR potential and confined by boundaries, whose separation could be varied at the cost of elastic energy. When the average number of particles is too small or too large for given $L$ for formation of the optimal bulk structure, some compromise must occur between the excess of the free energy associated with the structural deformations and the elastic energy cost due to adjusting the size of the compartment to the optimal structure of the confined fluid. We obtained exact results for a one-dimensional (1d) model in GCE and required mechanical equilibrium between the fluid-induced solvation force and the elastic force present when the system shrinks or expands. We found that when the equilibrium width of the empty container corresponded to the largest stress of the confined colloidal system, two significantly different system sizes were almost equally probable. The large size fluctuations are accompanied by absorption or evaporation of a whole cluster. Clearly, for fixed number of particles, such large size fluctuations are not possible. Hence, the permeability plays an important role in the case of elastic boundaries. In this work, we verify if in the case of fixed number of particles the bistability of the system size can still exist due to some other mechanism.

We present the model and the simulation methods in Sec. II. The density profiles are described and compared with the results obtained in the GCE in Sec. III. The mechanical properties are discussed in Sec. IV. We compare the dependence of pressure on density in the CE (fixed $N$ and varying $L$ ) and in the GCE for several fixed values of $L$ and varying $\langle N\rangle$. The dependence of pressure on the system size for fixed $N$ or $\langle N\rangle$ in the CE or GCE, respectively, is also discussed. In Sec. V, we consider elastic boundaries and compute the average system size as a function of $N$ for various elastic constants of the walls. For selected cases, the histograms for the fluctuating width of the system are presented. Section VI contains a short summary and concluding remarks.

\section{THE MODEL AND THE METHODS}

In this section, we briefly describe the model and the methods used for its analysis. More detailed descriptions of the model and the transfer matrix method used for finding the exact solutions of the model in the GCE can be found in Ref. 19.

\section{A. The model}

We consider a one-dimensional (1d) lattice model and assume an isotropic effective pair interaction potential of the SALR type. The ranges of the competing attractive and repulsive parts are chosen such that small clusters are formed for some range of thermodynamic variables. Namely, we assume that the nearest neighbors attract each other and the third neighbors repel each other, i.e., the effective pair interaction potential is

$$
V(\Delta x)= \begin{cases}-J_{1} & \text { for }|\Delta x|=1 \\ +J_{2} & \text { for }|\Delta x|=3 \\ 0 & \text { otherwise }\end{cases}
$$

where $J_{1}$ is the energy of attraction, $J_{2}$ is the energy of repulsion, the unit distance is the particle diameter $\sigma$, and $\Delta x$ is the distance between two sites of the lattice. Such a shape of the effective potential can be found for charged particles in solvents that induce short range attraction between the particles, e.g., for lysozyme molecules in water ${ }^{22}$ or for colloids in a solvent containing short-chain polymers. ${ }^{12}$

In Refs. 16-18, we assumed periodic boundary conditions and extensively investigated the spontaneous pattern formation of particles in the bulk. In order to study the effects of confinement on the self-assembled structures, we changed the boundary conditions of the model from periodic to rigid or elastic in Ref. 19. In the current study, we continue the investigation of the confined system with particles interacting via the pair potential given by Eq. (1). The confinement is assumed to be electrically neutral; hence, the interaction of the particles with the walls is short range. The Hamiltonians in the canonical and grand canonical ensembles take the forms,

$$
\begin{gathered}
U[\{\hat{\rho}\}]=\frac{1}{2} \sum_{x=1}^{L} \sum_{x^{\prime}=1}^{L} \hat{\rho}(x) V\left(x-x^{\prime}\right) \hat{\rho}\left(x^{\prime}\right)+h_{1} \hat{\rho}(1)+h_{L} \hat{\rho}(L) \\
(\text { fixed } N), \\
H[\{\hat{\rho}\}]=U[\{\hat{\rho}\}]-\mu \sum_{x=1}^{L} \hat{\rho}(x) \quad(\text { fixed } \mu),
\end{gathered}
$$

where $h_{1}$ and $h_{L}$ are the energies of the interactions between the confining walls and the particles located at the 1st and the $L$ th site, respectively, $\hat{\rho}(x)$ is the occupancy operator such that $\hat{\rho}(x)=1$ if the $x$-th site is occupied and $\hat{\rho}(x)=0$ otherwise, and $\mu$ is the chemical potential. The number of particles $N$ 
(fixed in the canonical ensemble) is given by

$$
N=\sum_{x=1}^{L} \hat{\rho}(x) .
$$

As in Ref. 19, we assume that the confinement can be either rigid or elastic. For rigid boundary conditions, the distance $L$ between the confining walls is fixed, while in the case of elastic walls, we assume that it may oscillate around $L_{0}$, which is the equilibrium width of an empty system. The Hamiltonian of the system with elastic boundary conditions is for the case of permeable walls,

$$
\mathcal{H}=H+k\left(L-L_{0}\right)^{2},
$$

where $k$ is the elastic constant, and for the fixed number of particles, the Hamiltonian takes the form,

$$
\mathcal{U}=U+k\left(L-L_{0}\right)^{2} .
$$

We set the energy of attraction $J_{1}$ as energy unit and introduce the following dimensionless variables

$$
\begin{aligned}
& T^{*}=k_{B} T / J_{1}, \quad \beta^{*}=J_{1} / k_{B} T, \quad J^{*}=J_{2} / J_{1}, \\
& h_{1}^{*}=h_{1} / J_{1}, \quad h_{L}^{*}=h_{L} / J_{1}, \quad \mu^{*}=\mu / J_{1}
\end{aligned}
$$

where $k_{B}$ is the Boltzmann's constant and $T$ is the temperature. From now on, we set $J^{*}=3, h_{1}^{*}=h_{L}^{*}= \pm 1$ and study the role of the temperature and the chemical potential or the number of particles. For the selected parameters, energy (2) takes the minimum when clusters composed of 3 particles are separated by 3 empty sites, and a cluster is attached to each boundary. Such an optimal structure is possible only for $L=2 N-3$.

\section{B. The computational method}

In order to find the exact solution of the model in the GCE for $T>0$ the transfer matrix method was applied. We have found exact expressions for the partition function and the local density. The details of the derivations are provided in the Appendix of Ref. 19. Here, we will present only the final formula for the partition function with a brief description of the notation. The partition function for the system of size $L=3 M+j$ with $M$ a natural number and $j=0,1,2$ is given by

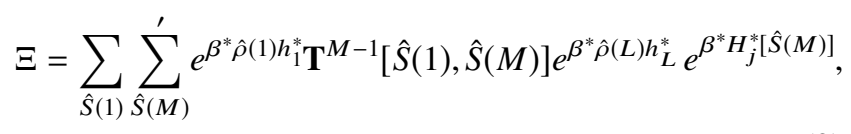

where $\hat{S}(n)=(\hat{\rho}(3 n-2), \hat{\rho}(3 n-1), \hat{\rho}(3 n))$ with $n=1, \ldots, M$ and $\mathbf{T}$ is the transfer matrix with the matrix elements defined as

$$
\mathbf{T}(\hat{S}(n), \hat{S}(n+1)) \equiv e^{-\beta^{*} H_{t}^{*}[\hat{S}(n), \hat{S}(n+1)]},
$$

where

$$
\begin{aligned}
& H_{t}^{*}[\hat{S}(n), \hat{S}(n+1)] \\
& \quad=\sum_{x=3 n-2}^{3 n}\left[-\hat{\rho}(x) \hat{\rho}(x+1)+J^{*} \hat{\rho}(x) \hat{\rho}(x+3)-\mu^{*} \hat{\rho}(x)\right] .
\end{aligned}
$$

$\sum_{\hat{S}(M)}^{\prime}$ and $H_{j}^{*}$ depend on $j$ and the rather lengthy expressions are provided in Appendix A. Having a formula for the partition function, one can derive exact expressions for the pressure and the local density. For more details as well as for asymptotic expressions of the exact solutions, see Ref. 19.

\section{The simulation methods}

In the current study, we compare the exact solution obtained in GCE with results of MC simulation in CE. In addition to the simulations in the $\mathrm{CE}$, we also carried out some runs in the GCE with the aim of cross-checking the consistency between the simulation codes and the numerical treatments based on the transfer matrix methods. The simulation procedures make use of the Metropolis criterion ${ }^{23}$ implemented for two kinds of MC steps: translations of one particle in the canonical ensemble and particle insertions or deletions in the grand canonical ensemble. For each step, a trial configuration is generated and it is accepted with probability: $\min [1, \exp (-\beta \Delta H)]$, where $\Delta H$ is the change of the Hamiltonian in the trial step. The trial configuration for a particle translation is generated by moving a randomly chosen particle to a randomly chosen empty site, which is equivalent to choose two sites of the lattice $x$ and $x^{\prime}$ so that $\hat{\rho}(x) \neq \hat{\rho}\left(x^{\prime}\right)$, and interchange their occupancy states so that $\hat{\rho}^{\text {trial }}(x)=\hat{\rho}\left(x^{\prime}\right)$ and $\hat{\rho}^{\text {trial }}\left(x^{\prime}\right)=\hat{\rho}(x)$. A MC step in the grand canonical simulations implies the insertion or deletion of one particle, this is achieved by choosing at random one site of the lattice, $x$, and generating the trial configuration by flipping its occupancy state from its current value $\hat{\rho}(x)$ to the trial value $\hat{\rho}^{\text {trial }}(x)=1-\hat{\rho}(x)$.

The computation of pressure from simulation of lattice models is usually carried out by means of the integration of the grand potential in the GCE, because the known relation for the canonical ensemble: $\beta p=-(\partial(\beta A) / \partial V)_{N, T}$ (where $A$ is the Helmholtz free energy and $V$ is the generalized volume) is hard to translate into an efficient numerical procedure due to the discreteness of the volume in lattice systems. We have found, however, that for our $1 \mathrm{~d}$ system, it is feasible to compute the pressure $p(N, L, T)$ in the CE, by an algorithm based on the discretization of the derivative of $A(N, L, T)$ with respect to the system size as

$$
p_{ \pm}(N, L, T)=\mp[A(N, L \pm 1, T)-A(N, L, T)] .
$$

The pressure, in the terms of the canonical partition function $Q(N, L, T)$, can be written as

$$
p_{ \pm}(N, L, T)= \pm \frac{1}{\beta} \ln \frac{Q(N, L \pm 1, T)}{Q(N, L, T)} .
$$

The two ways of discretization, $p_{+}$and $p_{-}$, lead to two different methods of computing the pressure, the virtual expansion, and the virtual contraction, respectively, from a direct analysis of the configurations from a simulation run at conditions $(N, L, T)$.

In the virtual expansion, an empty site is added at a randomly chosen position of the system. For a confined system of size $L$, there are $L+1$ possibilities of performing such an insertion, namely, $L-1$ cases where the inserted site is located between two sites of the system plus two insertions 
between the walls and the first or last site. Considering the $L+1$ possible ways of inserting an empty site on each of the microstates of the system of size $L$ with $N$ occupied sites (with $0 \leq N \leq L$ ), we get $L+1-N$ identical copies of each of the microstates of the system of size $L+1$ and $N$ occupied sites (see Appendix B). Let us denote by $\vec{\rho}_{L}$ a given configuration of the system with $N$ particles and $L$ sites, with potential energy given by $U\left(\vec{\rho}_{L}\right)$. If we define $\vec{\rho}_{L+1}\left(\vec{\rho}_{L}, k\right)$ as the configuration with $N$ particles of a system with $L+1$ sites built from $\vec{\rho}_{L}$ by inserting a site at position $k$ and denote by $\sum_{\vec{\rho}_{L}}$ the sum over all possible microstates of this system with $N$ particles and $L$ sites, then we can write Eq. (13) as

$$
\begin{aligned}
p_{+}(N, L, T) & =\frac{1}{\beta} \ln \frac{\sum_{\vec{\rho}_{L}} \exp \left[-\beta U\left(\vec{\rho}_{L}\right)\right] \sum_{k=0}^{L} \exp \left[-\beta U\left(\vec{\rho}_{L+1}\left(\vec{\rho}_{L}, k\right)\right)+\beta U\left(\vec{\rho}_{L}\right)\right]}{(L+1-N) \sum_{\vec{\rho}_{L}} \exp \left[-\beta U\left(\vec{\rho}_{L}\right)\right]} \\
& =k_{B} T \ln \left\langle\frac{L+1}{L+1-N} \exp \left[-\beta \Delta U_{i n s}\right]\right\rangle_{L},
\end{aligned}
$$

where $\langle\cdot\rangle_{L}$ is the average value of $\cdot$ when sampled on a system of size $L$, and $\Delta U_{\text {ins }}$ is the difference between the energies of the systems with $L+1$ and $L$ sites. Analogously, a formula for pressure $p_{-}$computed by the virtual contraction scheme can be derived

$$
p_{-}(N, L, T)=-k_{B} T \ln \left\langle\frac{L-N}{L} \exp \left[-\beta \Delta U_{d e l}\right]\right\rangle_{L},
$$

where $\Delta U_{d e l}=U\left(\vec{\rho}_{L-1}\right)-U\left(\vec{\rho}_{L}\right)$ is the variation of energy when a configuration $\vec{\rho}_{L-1}$, of $N$ particles and $L-1$ sites is generated by eliminating one of the empty sites from the configuration $\vec{\rho}_{L}$ of a system with $N$ particles and $L$ sites. The virtual contraction method is inefficient at high densities; therefore, we used it only for verification of the results obtained via the virtual expansion method, since by construction we expect

$$
p_{+}(N, L, T)=p_{-}(N, L+1, T) .
$$

In order to calculate properties of the system with elastic boundary conditions described by the Hamiltonian $\mathcal{U}$ given in Eq. (6), one needs to perform two additional types of MC steps. The first one is the intercalation of an empty site into a randomly chosen place of the system; the second one is the removal of a randomly chosen empty site of the system. The acceptance probability of the first move for system with $L$ sites and $N$ particles is

$$
\mathcal{A}(L+1 \mid L)=\min \left\{1, \exp [-\beta \Delta \mathcal{U}] \frac{L+1}{L+1-N}\right\},
$$

where $\Delta \mathcal{U}$ is the change of the energy after the size modification. Analogously, the probability of acceptance of the move in which an empty site is removed is given by

$$
\mathcal{A}(L-1 \mid L)=\min \left\{1, \exp [-\beta \Delta \mathcal{U}] \frac{L-N}{L}\right\} .
$$

Notice that this procedure resembles a lattice version of isothermal-isobaric $(N p T)$ simulation, in which the energy term introduced through the elastic force plays the role of the external field.

\section{DISTRIBUTION OF PARTICLES BETWEEN RIGID WALLS}

In this section, we consider a system containing $N_{0}$ particles between rigid walls separated by a fixed distance $L$. The main question is how the particles self-assemble if $N_{0}$ is such that the equilibrium bulk structure is not possible, but it is big enough to form clusters. The distribution of particles for fixed $N_{0}$ will be compared with the distribution of particles in the open system, where the number of particles $N$ fluctuates in such a way that $\langle N\rangle=N_{0}$.

In Fig. 1, the density profiles obtained by the $\mathrm{MC}$ simulations in the $\mathrm{CE}$ are compared with the exact results obtained in the GCE by the transfer matrix method described in Ref. 19. We chose $L=50, T^{*}=0.3$, and several values of $N_{0}$. In each case, the chemical potential in the GCE was fixed to the value that corresponds to $\langle N\rangle=N_{0}$. We used the exact expression for density as a function of the chemical potential that was obtained in Ref. 19. For $L=51$, the optimal number of clusters for the considered range of $\mu^{*}$ is 9 (hence $N=27$ ), since the sequence of three occupied sites followed by three empty sites can be formed, with two clusters adsorbed at the attractive surfaces. The energy for such a structure assumes a minimum (there are as many attracting pairs as possible with no repulsion). For $L=50$, only a small defect in the ordered structure occurs. We can see a very good agreement between the two ensembles for small as well as for large number of particles. For $N=24$ corresponding to eight clusters, however, the number of maxima in the GCE is larger than in the CE. This result is even more surprising when we consider the fluctuation of the number of particles in the GCE (Fig. 2). One can see that the largest discrepancy between the density profiles does not occur for the largest fluctuation of the number of confined particles in the GCE.

In order to understand why the distributions of the particles in the two ensembles are different when one cluster in the CE "is missing," let us consider the ground state (GS), $T^{*}=0$. The microstates present in the GS for $L=15$ and $N=5,6,7$ are shown in Fig. 3. The GS in Fig. 3 shows that even small fluctuations of the number of particles-addition of one particle in our case — can lead to a significant change in 

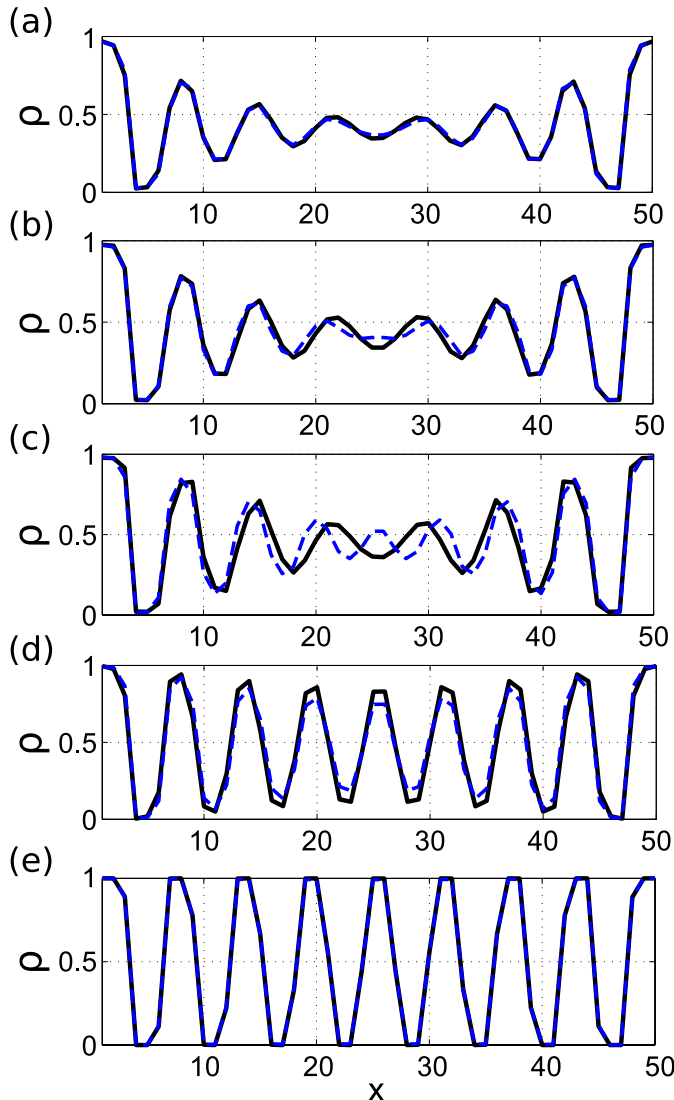

FIG. 1. Comparison of the GCE (dashed line) and the CE (solid line) density profiles for $L=50$ at $T^{*}=0.3$ and $N=22$ or $\mu^{*}=-0.33904$ (a), $N=23$ or $\mu^{*}=-0.21127$ (b), $N=24$ or $\mu^{*}=-0.02632$ (c),$N=25$ or $\mu^{*}=0.22159$ (d), $N=26$ or $\mu^{*}=1.08857$ (e). Repulsion to attraction ratio $J^{*}=3$ and attractive walls with $h_{1}^{*}=h_{L}^{*}=-1$ are considered for all the cases.

the distribution of the particles. This is the case when $\langle N\rangle$ is a multiple of 3 , and there is a free space for an extra cluster (with no cluster-cluster repulsion). When one additional particle enters the system, the energy change is $J^{*}-1$ when one of the clusters grows to contain 4 particles, or 0 , when the new particle is sufficiently far from the clusters, or one of the clusters together with the new particle form two clusters composed of two particles. In Fig. 3, bottom row, the two latter cases are shown. The states shown in Fig. 3 are energetically favorable for $J^{*}>1$. Thus, in the GCE with $\langle N\rangle=6$, such

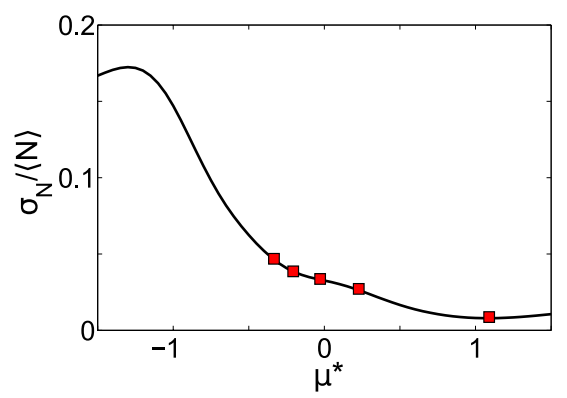

FIG. 2. The standard deviation of the number of particles $\sigma_{N}$ divided by the average number of particles $\langle N\rangle$ for $L=50, T^{*}=0.3, J^{*}=3$, and attractive walls with $h_{1}^{*}=h_{L}^{*}=-1$. The red squares indicate the values of the chemical potential taken for the density profiles in Fig. 1.

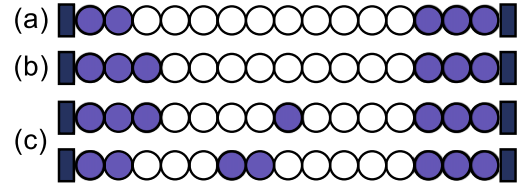

FIG. 3. Typical microscopic states present in the GS of the model with $J^{*}=3$, attractive walls with $h_{1}^{*}=h_{L}^{*}=-1$ and $L=15$ in the CE with $N=5$ (a), $N=6$ (b), and $N=7$ (c). For $N=5,7$, the GS is degenerate. Blue color denotes the occupied sites, while white the empty sites.

microstates will appear quite often. As a result, an additional maximum in the average density profile occurs.

The above simple considerations show that spatial distribution of particles in the CE and GCE can be qualitatively different. This qualitative difference is not present for the largest fluctuation of the number of particles in the GCE. Even a small fluctuation of the number of particles can lead to a change of the number of clusters, because the sizes of the clusters can fluctuate, especially when $N$ is not a multiple of 3 . When $\langle N\rangle$ is small enough, the additional clusters can occupy the empty space and no inter-cluster repulsion appears.

The system size for which the described qualitative difference between the ensembles is still present can be thousands of times larger then the particle diameter and it increases upon cooling. Such effect follows from the very large correlation length $\xi$ between the particles in this model. ${ }^{16}$ If $L \gg 2 \xi$, we do not expect the ensembles to be different, because in such a case the density profile in the middle of the slit is uniform. Thus, the ensembles are equivalent in thermodynamic limit.

On the other hand, we have observed differences between the ensembles for very low densities, too small for clustering. In this limit, the differences follow from the high density fluctuations in the GCE. ${ }^{20}$ Interestingly, when two clusters can be formed in the system, we have obtained results very similar to those shown in Fig. 1(b) of Ref. 20, but with two particles replaced by two clusters.

\section{EQUATION OF STATE IN A SYSTEM CONFINED BY RIGID WALLS}

In this section, we compute pressure for fixed number of confined particles $N_{0}$ as a function of the distance between the confining surfaces $L$. From these results, we obtain the pressure as a function of density, $p(\rho)$, for given $N_{0}$. For comparison, we present $p(\rho)$ calculated exactly in the GCE by the transfer matrix method described in Ref. 19. In the GCE, we consider fixed $L$ and $\mu$ and calculate $p(\mu)$ and $\rho(\mu)$ to obtain $p(\rho)$ for given $L$. The shape of $p(\rho)$ in the GCE depends on the commensurability between $L$ and the period of the energetically favorable structure. We shall compare the results obtained in the CE for fixed $N_{0}$ with the $p(\rho)$ lines obtained in the GCE for six system sizes $L$.

In Fig. 4, the results for the reduced pressure as a function of the average density for CE and GCE are presented. Note the discrepancy between the $\mathrm{CE}$ and GCE for $\rho \approx 0.55$, where the periodically ordered clusters consisting of 3 particles are separated by 3 empty sites. In the GCE, $p(\rho)$ increases 

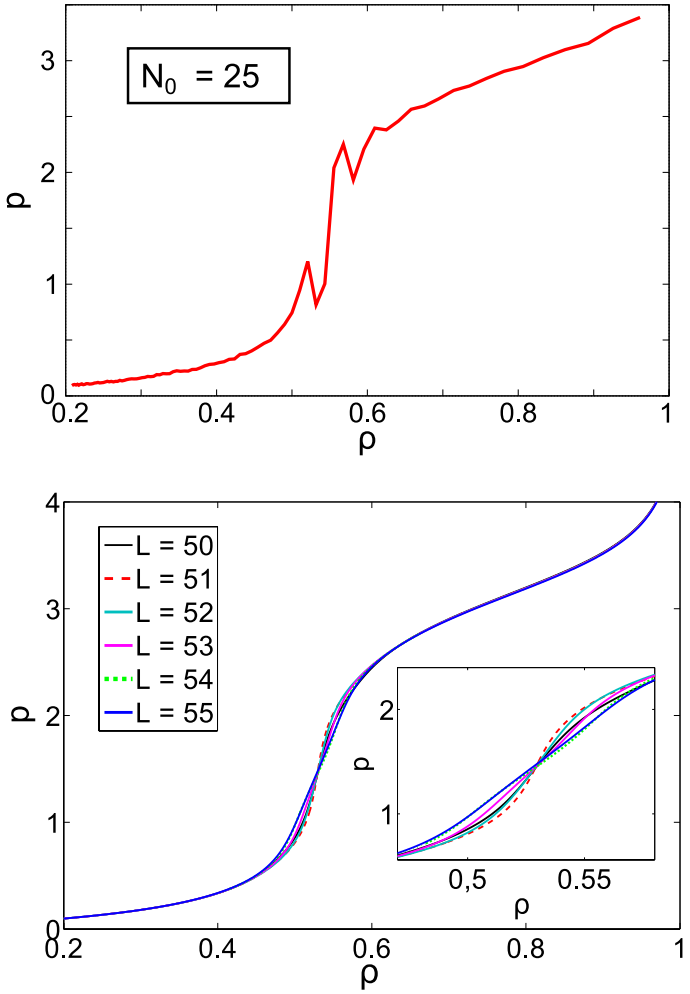

FIG. 4. Reduced pressure as a function of density for $J^{*}=3, T^{*}=0.5$, and attractive walls $\left(h_{1}^{*}=h_{L}^{*}=-1\right)$. Upper panel: Canonical Monte Carlo (CMC) simulation for the number of particles $N_{0}=25$ and different system sizes $L$, obtained via virtual insertion method. Lower panel: GCE exact results for pressure vs. density for different system sizes, $L=50,51,52,53,54,55$.

monotonically, although for $\rho \approx 0.55$, the slope is very large, and an inflection point is present. We should stress that in the $\mathrm{CE}, N_{0}$ is fixed and the density changes because of the change of $L$. In contrast, in the GCE $L$ is fixed, and the density changes because $\mu$, and as a result $\langle N\rangle$, changes. In the GCE, there are different branches of $p(\rho)$ for different $L$. One may interpret the nonmonotonic $p(\rho)$ in the $\mathrm{CE}$ as a consequence of the jumps between the different branches of $p(\rho)$ in the GCE for $L$ and $L-1$.

In order to separate the effect of the fluctuation of the number of particles and the effect of the method by which the density changes, we compare the $p(L)$ curves in the CE with $N_{0}$ particles and in the GCE with $\langle N\rangle=N_{0}$. In Fig. 5, the pressure is shown as a function of $L$ for the CE with $N_{0}=21$ and for GCE with $\langle N\rangle=21$. The GCE curve was obtained by finding for each system size $L$ the value of the chemical potential $\mu_{0}$ such that $\rho\left(\mu_{0}\right) \approx N_{0} / L$. We used the exact expression for density obtained in Ref. 19. For such chemical potential, the pressure was computed from the approximate formula $\beta p$ $=-\ln \Xi\left(\mu_{0}, L+1, T\right)+\ln \Xi\left(\mu_{0}, L, T\right)$, which is the 1d lattice version of the standard expression $p=-(\partial \Omega / \partial V)_{\mu, T}$. We also present the density profiles for $L=35,36,37,38$, where $p$ changes rapidly in a nonmonotonic way. In the case of attractive surfaces, the periodic structure where three occupied sites are separated by three empty sites is possible for $L=39$ and corresponds to seven clusters. For $L<39$, either the clusters are bigger, or the distances between them are smaller. In both cases, the repulsion between the particles is present,

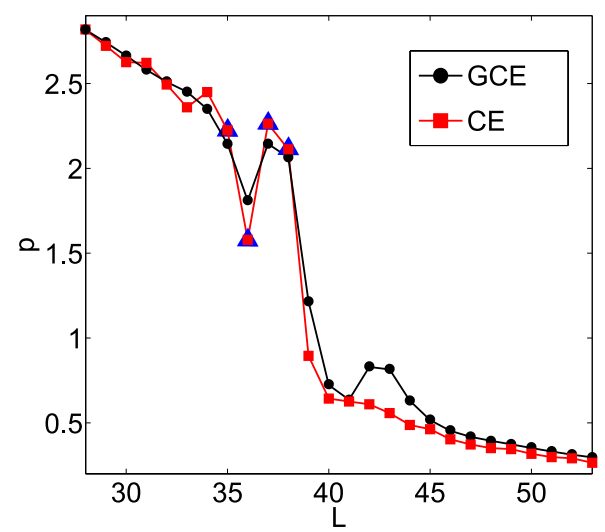

(a)

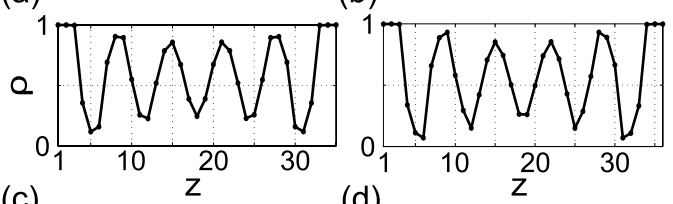

(c)

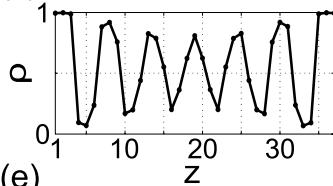

(d)

(e)
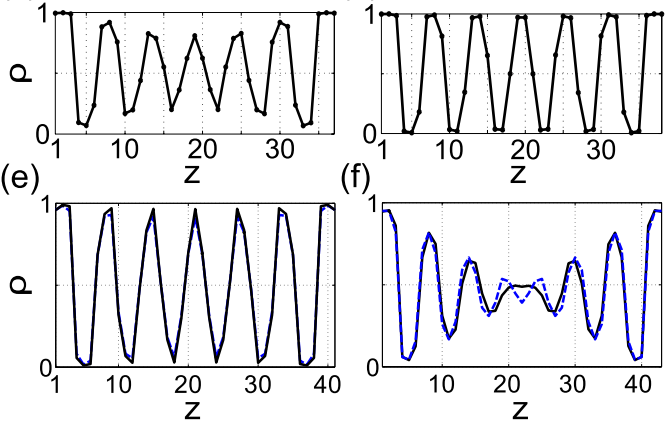

(f)

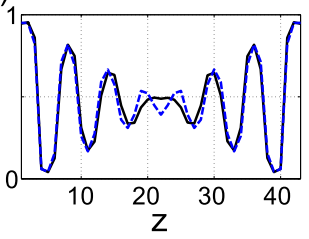

FIG. 5. Upper panel: reduced pressure as a function of the system size $L$ for $J^{*}=3$ and $T^{*}=0.5$ in the case of attractive walls $\left(h_{1}^{*}=h_{L}^{*}=-1\right)$. Red curve with squares: CMC simulation for 21 particles. Black curve with bullets: exact results in the GCE with the average number of particles equal to 21 . Lower panels: ((a)-(d)) CE density profiles in systems with $L=35$ (a), $L=36$ (b), $L=37$ (c), and $L=38$ (d) (indicated on the $p(L)$ plot as blue triangles). Panels ((e) and (f)): comparison of the density profiles in CE (solid line) and GCE (dashed line) for $L=42$ (e) and $L=43$ (f).

and pressure increases. In Fig. 5, we can see six clusters for $L<37$ and seven clusters for $37 \leq L \leq 42$. Note that the nonmonotonic dependence of $p$ on $L$ corresponds to the jump of the number of clusters. The unusual increase of pressure in the expanding system results from the transition to a larger number of smaller clusters. The clusters repel each other for $L<39$. Upon increase of the system size from $L=38$ to $L=39$, the separation between the clusters becomes large enough to put the clusters at the separations larger than the range of repulsion, and the pressure drops.

It is interesting that although both the average densities and density profiles for $L=42$ in the two ensembles are the same, the pressure is different. The reason is that the pressure depends not on the values of the thermodynamic potentials at a given state, but on their change and as can be seen on panel (f) of Fig. 5, for $L=43$, the profiles differ significantly.

We conclude that the mechanical properties of a confined self-assembling system depend significantly on whether the system expands for given number of particles, or the separation between the system boundaries is fixed, and the number of particles decreases due to a change of the chemical potential. 

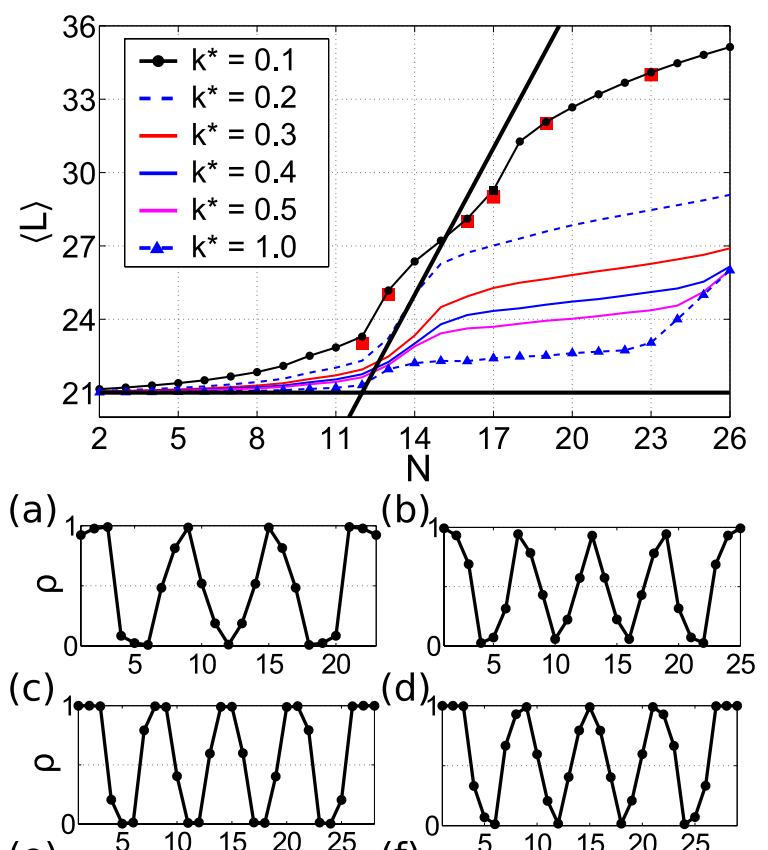

(d)
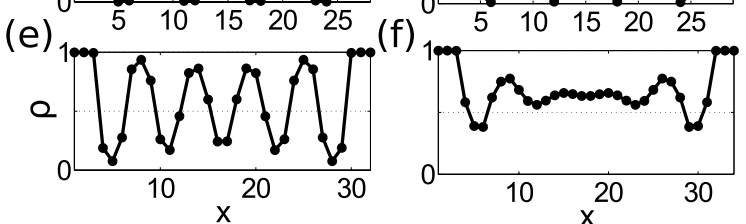

FIG. 6. Average system size as a function of the number of particles for different spring constants $k^{*}$. The walls are attractive, $h_{L}^{*}=h_{1}^{*}=-1, J^{*}=3$, $T^{*}=0.5$, and $L_{0}=21$. Thick solid black lines, $L=L_{0}$ and $L=2 N-3$, correspond to rigid walls and to walls fully adapting to the optimal structure, respectively. In the panels ((a)-(f)), the density profiles for the number of particles and the system size marked by the red squares along the black solid curve ( $k^{*}=0.1$ ) are shown. (a) $L=23$ and $N=12$, (b) $L=25$ and $N=13$, (c) $L=28$ and $N=16$, (d) $L=29$ and $N=17$, (e) $L=32$ and $N=19$, (f) $L=34$ and $N=23$.

In both cases, we can have the same change of density, but different changes of pressure. The unusual increase of pressure upon system expansion is found only in the case of fixed (average) number of particles and is connected with a significant structural reorganization.

\section{THE CASE OF ELASTIC BOUNDARIES}

In this section, we assume that the number of particles $N$ is fixed, while the separation between the system boundaries is not fixed and can fluctuate around $L=L_{0}$. The change of the wall separation is associated with the energy cost $\Delta U^{*}=k^{*}\left(L-L_{0}\right)^{2}$. Here, $k^{*}$ denotes the elastic constant in units $J_{1} / \sigma^{2}$. In Fig. 6, the average system size $\langle L(N)\rangle$ as a function of the number of particles $N$ is presented. The confining surfaces are kept at the separation $L$ by the spring that is at rest for $L_{0}=21$. We assume attractive walls and $T^{*}=0.5$. In a system with rigid boundary conditions, attractive walls and $L=21$, the periodic structure made of 12 particles is energetically favorable. Thus, for $N \leq 12$ only for small values of the spring constant (e.g., $k^{*}=0.1$ ), significant deviations of the average system size from the reference value are present. For $N>12$ the internal stress of the fluid competes with the elastic forces and $\langle L\rangle>L_{0}$ even for $k^{*}=1$.

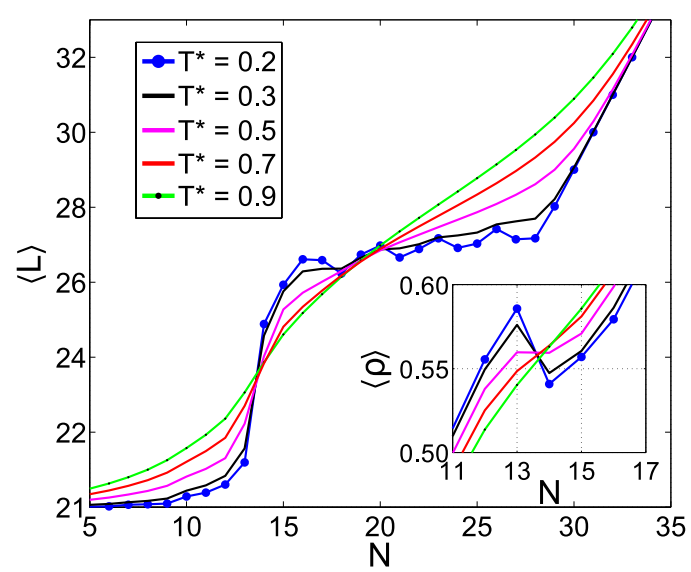

FIG. 7. Average system size as a function of the number of particles for different temperatures. Attracting walls assumed $\left(h_{1}^{*}=h_{L}^{*}=-1\right), J^{*}=3$, spring constant $k^{*}=0.2$ and $L_{0}=21$. The inset presents the average density $\langle\rho\rangle$ $\equiv N /\langle L\rangle$ as a function of the number of particles.

We can distinguish two limiting cases: (i) stiff spring, where the system size saturates and the particles become densely packed when $N$ increases, and (ii) soft spring, where the average system size increases with increasing $N$, and the clusters are separated by empty sites. If $k^{*}$ is small enough, then the slope of $\langle L(N)\rangle$ increases when a new cluster made of 3 particles is introduced to the system (see panels (a)-(f) of Fig. 6). On the other hand, for larger values of $k^{*}$, the elastic forces are stronger, and the system tends to modify the structure of the fluid rather than the system size. Between the two limiting cases, there is an interesting region where the elastic and the solvation forces are comparable and compete.

In Fig. 7, we show $\langle L(N)\rangle$ for $L_{0}=21$ and $k^{*}=0.2$ for several temperatures. At low temperatures, three regimes with significantly different slopes of the lines $\langle L(N)\rangle$ can be distinguished. For $N \lesssim 12$, the slope of the line $\langle L(N)\rangle$ is small, because in this case the separation between the clusters ensures no repulsion between them. For $12 \lesssim N \lesssim 15$, the system expands significantly upon addition of particles, because for $N=14$ an additional cluster appears. In this region, the average size of the clusters and the distance between them is 3 . For $N \gtrsim 15$, the slope is small again. Here, elastic stress dominates and the clusters average size increases until the system becomes densely packed. Note that in this region and at low $T$, before the system gets filled with particles, we obtain an oscillatory dependence of $\langle L(N)\rangle$ on $N$, with the minima occurring when the number of particles is a multiple of 3 . Note also that for a given $N,\langle L\rangle$ increases with temperature except from $13<N<21$, where for $L=L_{0}$ the density is between the density of the periodic and the closely packed structures. We verified that the anomalous contraction of the heated system is no longer observed at high T. Finally, note that there is some similarity of the shapes of the $\langle L(N)\rangle$ and $p(\rho)$ lines (Figs. 7 and 4).

The average wall separation and the average density profile give insufficient information about the system behavior. In Fig. 8, we present histograms for the wall separation. Two cases can be distinguished - a single maximum in the probability of the appearance of the wall separation $L$ and a bistability with two maxima in this probability, separated by $\Delta L=3$. 

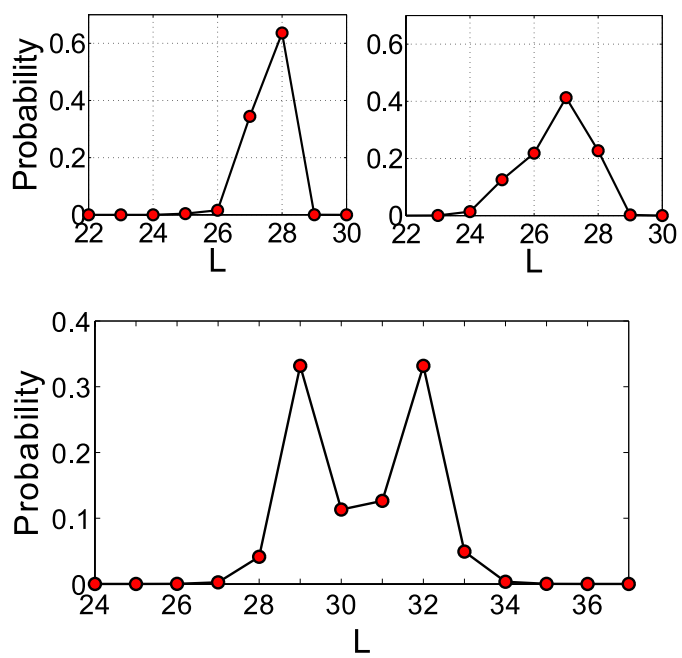

FIG. 8. First row: the histograms for the wall separation in the case of elastic boundaries with the spring constant $k^{*}=0.2, N=16, L_{0}=21, J^{*}=3$ and attractive walls $\left(h_{1}^{*}=h_{L}^{*}=-1\right)$ for $T^{*}=0.2$ (left panel), and $T^{*}=0.5$ (right panel). Second row: the histogram for the wall separation in the case of elastic boundaries with the spring constant $k^{*}=0.06607, J^{*}=3, T^{*}=0.5, N=17$, $L_{0}=19$, attractive walls $\left(h_{1}^{*}=h_{L}^{*}=-1\right)$.

In order to understand the energetics associated with the bistability, let us consider the GS for $L_{0}=10$ and $N=7$. The microstates shown in Fig. 9 correspond to the same energy of the confined system (6), when $J^{*}=3$ and $k^{*}=2 / 9$. Two different system sizes in the GS can occur when $N$ is not a multiple of 3 , and for $L=L_{0}$ an intra-cluster repulsion is present. The expansion is associated with a simultaneous increase of elastic energy of the walls and decrease of the internal energy of the particles, when the separations between
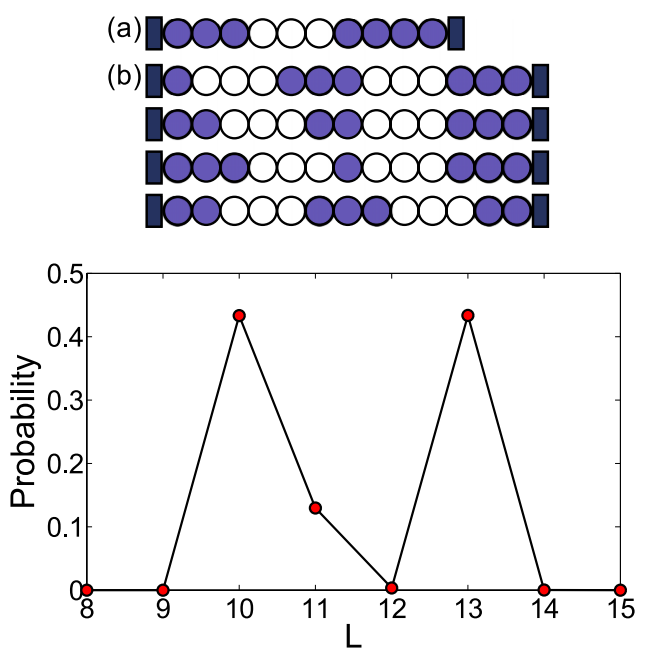

FIG. 9. Upper panel: microstates in the GS of a system consisting of 7 particles with the repulsion to attraction ratio $J^{*}=3$, confined by attractive walls $\left(h_{1}^{*}=h_{L}^{*}=-1\right)$ on a spring with the spring constant $k^{*}=2 / 9$ that is at rest for $L_{0}=10$. In panel (a), $L=L_{0}$, while in panel (b), $L=L_{0}+3$. Blue color denotes the occupied sites, while white the empty sites. Note that the microstates symmetric to those also have the same energy; hence for $L=10$, there are 2 different microstates with the same energy and for $L=13$, there are 6 . These microstates correspond to the maxima of the probability shown in the lower panel, in which we present the histogram for the wall separation in the above system in the case of $T^{*}=0.2$ and elastic boundaries with the spring constant $k^{*}=2 / 9+0.0244135$. them are such that the repulsion is absent. Each microstate in Fig. 9 occurs with the same probability, but because of the difference in the degeneracy for $L=L_{0}$ and $L=L_{0}+3$, the probability ratio for the two lengths is $p\left(L_{0}+3\right) / p\left(L_{0}\right)=3$. To estimate the spring constant leading in the above example to equal probability of $L_{0}$ and $L_{0}+3$ for low $T^{*}$, we take into account only the microstates shown in Fig. 9 and require that $\exp \left(-\beta^{*}\left(-5+J^{*}\right)\right)=3 \exp \left(-\beta^{*}\left(-4+9 k^{*}\right)\right)$ (see (6) for $L$ $=L_{0}, L_{0}+3$ ). For $T^{*}=0.2$, we obtain $k^{*} \approx 2 / 9+0.0244136$ in very good agreement with the results of simulations shown in Fig. 9.

\section{SUMMARY AND CONCLUDING REMARKS}

We have studied the effect of various constraints on colloidal self-assembly in thermodynamic states that correspond to self-assembly into small clusters separated by voids. In our model system, small clusters with no intra-cluster repulsion yield a negative contribution to the internal energy and do not interact with one another if the separation between them is larger than the range of repulsion. The positions, the size, and the number of clusters can fluctuate and different deformations of the bulk structure are possible in confinement. In order to determine the role of constraints imposed on the number of particles and/or the size of the system, we have compared density profiles, equations of state, and effects of elastic boundary conditions.

\section{A. Structure}

If the number of particles in the CE is too small for formation of the bulk structure, then one more cluster can be present in the GCE despite the same average number of particles (Fig. 1). Interestingly, the different number of clusters in the two ensembles is observed for thermodynamic states that do not correspond to the maximum of the fluctuation of the number of particles. This is because the fluctuations of the number of particles are coupled with the fluctuations of the size of the clusters. Even a small increase of the number of particles in the GCE together with the splitting of the clusters can lead to formation of a larger number of smaller clusters. The qualitative difference between the two ensembles occurs for quite large number of particles. This behavior is different than in simple fluids, where the difference between the two ensembles was observed for very small number of particles confined in very small pores. ${ }^{24,25}$

\section{B. Equation of state}

In the bulk, the isotherms $p(\rho)$ do not depend on the way in which the variation of density is attained. In the confined inhomogeneous systems, it is no longer the case: $D$ ifferent curves $p(\rho)$ are obtained when the size of the system changes with fixed number of particles or when the number of particles changes at fixed system size.

The shape of the $p(\rho)$ curve obtained in the GCE with fixed $L$ depends significantly on $L$, or more precisely on the commensurability of $L$ and the period of the ordered structure (Fig. 4). In the CE with fixed $N$, we have obtained anomalous 
decrease of pressure for increasing density for small density intervals below and above the density of the equilibrium bulk structure (Fig. 4). Inspection of density profiles shows that the anomalous increase of pressure for increasing system size with fixed number of particles is accompanied by increased number of clusters (the larger cluster splits) (Fig. 5). Recall that at short separations, the clusters repel each other, and this leads to the increase of pressure. The pressure rapidly drops when $L$ further increases and the clusters do not repel one another any more. In order to check if the anomalous dependence of pressure on density follows from the fixed number of particles or from the process by which the density varies, we computed the $p(L)$ curve in the GCE with fixed average number of particles. We have obtained similar curves in the two ensembles except for large slits. In both ensembles, the anomalous increase of pressure for increasing $L$ is associated with the increasing number of clusters. For some large $L$, the number of clusters increases with the system size in the GCE but not in the CE (Figs. 5(e) and 5(f)). In this case, a maximum in the $p(L)$ curve is present only in the GCE.

\section{Bistability in elastic confinement}

If the width of the slit can vary, then the system tends to equilibrate the competing solvation and elastic forces. We have found that the equilibrium size of the system is not always unique. In Ref. 19, we observed a bistability in a system confined by elastic walls with permeable walls (fixed $\mu)$. Two different system sizes can be equally probable: one with expanded and the other one with compressed boundaries. The size fluctuations are accompanied by an absorption or an evaporation of a whole cluster. In the case of impervious walls (fixed $N$ ), a bistability exists too. In both cases, the origin of the bistability is the change of the number of clusters, but the mechanisms which lead to the change are different. The number of clusters can fluctuate for fixed number of particles when the state with a smaller number of bigger clusters and the state with a larger number of smaller clusters are equally probable. When the intra-cluster repulsion in large clusters competes with the elastic energy of stretched boundaries, the clusters can split and separate. The difference between the two equilibrium widths of the system is equal to the period of the bulk structure in the case of permeable walls and to half the period of the bulk structure when the number of particles is constrained. Since in our $1 \mathrm{~d}$ system the energy barrier is $\sim k_{B} T$, spontaneous changes of the system size may be induced by thermal fluctuations.
Our results show that different anomalies in the confined inhomogeneous systems occur when the release of some constraint or a change of the thermodynamic state leads to a change of the number of clusters. In particular thermodynamic states, the structure and anomalies depend qualitatively on the ability of the system to interchange particles with its surroundings.

Let us stress the difference between the confined simple fluids and the SALR systems. Packing effects of molecules or clusters play important role in both cases, especially for the solvation force that exhibits oscillatory decay on the corresponding length scale in each case. However, the clusters can split or merge, unlike the molecules. This leads to qualitative differences between the ensembles, anomalies in the $p(\rho)$ and the $\langle L(N)\rangle$ curves, and the bistability of the system size that in simple fluids are absent.

The patterns emerging in the colloidal and amphiphilic self-assembly are very similar. ${ }^{26-28}$ The clusters or layers composed of particles are distributed in space in a similar way as micelles or bilayers composed of amphiphilic molecules. Based on the similarity between the two types of selfassembly, we can expect that our results may also concern amphiphilic systems such as surfactants or lipids in water and block copolymers, at least on a qualitative level. Similarly, magnetic systems with competing interactions ${ }^{29-32}$ may have very similar properties in confinement.

\section{ACKNOWLEDGMENTS}

J.P. acknowledges the financial support by the National Science Center under Contract Decision No. DEC2013/09/N/ST3/02551. N.G.A. acknowledges the support from the Dirección General de Investigación Científica y Técnica (Spain) under Grant No. FIS2013-47350-C5-4-R. A.C. acknowledges the financial support by the National Science Center Grant No. 2012/05/B/ST3/03302. J.P. received funding for the preparation of the doctoral dissertation from the National Science Center in the funding of PhD scholarships on the basis of the Decision No. DEC-2014/12/T/ST3/00647.

\section{APPENDIX A: THE EXPRESSIONS FOR $H_{j}^{*}$ AND $\Sigma_{\hat{\mathcal{S}}(M)}^{\prime}$}

The $H_{j}^{*}$ contains the pair interactions between the particles at the sites of the $M$-th box, as well as the interactions between the particles at the sites labeled $3 M+1$ and $3 M+2$ (if they exist for given $L$ ),

$$
H_{j}^{*}[\hat{S}(M)]= \begin{cases}-\left(\sum_{i=0}^{1} \hat{\rho}(3 M-i) \hat{\rho}(3 M-i-1)\right)-\mu^{*}\left(\sum_{i=0}^{2} \hat{\rho}(3 M-i)\right), & \text { if } j=0 \\ -\left(\sum_{i=0}^{2} \hat{\rho}(3 M+1-i) \hat{\rho}(3 M-i)\right)+J^{*} \hat{\rho}(3 M-2) \hat{\rho}(3 M+1) & \text { if } j=1 \\ -\mu^{*}\left(\sum_{i=0}^{3} \hat{\rho}(3 M+1-i)\right), & \\ -\left(\sum_{i=0}^{3} \hat{\rho}(3 M+2-i) \hat{\rho}(3 M+1-i)\right)+ & \text { if } j=2 \\ J^{*}\left(\sum_{i=0}^{1} \hat{\rho}(3 M-2+i) \hat{\rho}(3 M+1+i)\right)-\mu^{*}\left(\sum_{i=0}^{4} \hat{\rho}(3 M+1-i)\right) . & \end{cases}
$$


Whereas $\sum_{\hat{S}(M)}^{\prime}$ denotes

$$
\sum_{\hat{S}(M)}^{\prime}= \begin{cases}\sum_{\hat{S}(M)} & \text { if } j=0 \\ \sum_{\hat{S}(M)} \sum_{\hat{S}(M)} \sum_{\hat{\rho}(3 M+1)} \sum_{\hat{\rho}(3 M+2)} & \text { if } j=1 \\ & \text { if } j=2 .\end{cases}
$$

\section{APPENDIX B: MICROSTATES OBTAINED BY THE VIRTUAL EXPANSION OF THE SYSTEM}

We consider $N$ indistinguishable particles and $L$ lattice sites. Each site can be empty or occupied by one particle, thus there are $\left(\begin{array}{l}L \\ N\end{array}\right)$ distinguishable microstates. We will show that the virtual expansion procedure of building configurations of the system with $L+1$ sites by inserting an empty site at a random position in a system with $L$ sites is not biased by the insertion procedure. Let us consider two sets of particle configurations. The elements of the first set are the microstates of a system of size $L$ with $N$ occupied sites with a distinguished position in which a new site can be inserted. Since the new site can be inserted in $L+1$ places, the first set contains $(L+1)\left(\begin{array}{l}L \\ N\end{array}\right)$ different elements. In the second set, each of the elements corresponds to one of the microstates of a system of $L+1$ sites with $N$ occupied sites and with one of its $L+1-N$ empty sites marked as removable. The number of elements of the second set is $(L+1-N)\left(\begin{array}{c}L+1 \\ N\end{array}\right)$. Because $(L+1)\left(\begin{array}{c}L \\ N\end{array}\right)$ $=(L+1-N)\left(\begin{array}{c}L+1 \\ N\end{array}\right)$, the two sets are equinumerous. We define a one-to-one correspondence between the elements of the two sets by identifying the location of the insertion point in an element of the first set with the location of the removable site in the element of the second set and by requiring that the same sites are occupied. Note that each microstate of the system with $L+1$ sites can be obtained in $L+1-N$ ways from the elements of the second set by removing the mark "removable." Because of the one-to-one correspondence between the elements of the two sets, each microstate of the system with $L+1$ sites is obtained $L+1-N$ times by the above procedure.

It follows that the proposed procedures of building configurations of the system with $L+1$ sites by inserting an empty site at a random position on the configurations of a system with $L$ sites are not biased by the insertion procedure. The same lack of bias applies in the reverse procedure.
For an illustration, let us consider $L=2$ and $N=1$. There are 2 microstates, [1,0] and [0,1]. After insertion of an empty site in 3 possible places, we obtain from the first microstate $[0,1,0],[1,0,0],[1,0,0]$ and from the second microstate $[0,0,1],[0,0,1],[0,1,0]$. One can easily see that after this procedure, we obtained each microstate in the system of size $L+1=3$ containing $N=1$ particle $L+1-N=2$ times.

${ }^{1}$ R. Evans, J. Phys.: Condens. Matter 2, 8989 (1990).

${ }^{2}$ J. N. Israelachvili, Intermolecular and Surface Forces, 3rd ed. (Academic Press, 2011).

${ }^{3}$ P. Kowalczyk, A. Ciach, and A. Neimark, Langmuir 24, 6603 (2008).

${ }^{4}$ P. Kékicheff and H. K. Christenson, Phys. Rev. Lett. 63, 2823 (1989).

${ }^{5}$ D. A. Antelmi, P. Kékicheff, and P. Richetti, J. Phys. II 5, 103 (1995).

${ }^{6}$ M. Tasinkevych and A. Ciach, Phys. Rev. E 60, 7088 (1999).

${ }^{7}$ A. Ciach, Progr. Colloid Polym. Sci. 129, 40 (2004).

${ }^{8}$ A. J. Archer, Phys. Rev. E 78, 031402 (2008).

${ }^{9}$ A. Imperio and L. Reatto, Phys. Rev. E 76, 040402 (2007).

${ }^{10}$ S. Perkin and J. Klein, Soft Matter 9, 10438 (2013).

${ }^{11}$ A. I. Campbell, V. J. Anderson, J. S. van Duijneveldt, and P. Bartlett, Phys. Rev. Lett. 94, 208301 (2005).

${ }^{12}$ A. Stradner, H. Sedgwick, F. Cardinaux, W. Poon, S. Egelhaaf, and P. Schurtenberger, Nature 432, 492 (2004).

${ }^{13}$ A. Imperio and L. Reatto, J. Phys.: Condens. Matter 16, S3769 (2004).

${ }^{14}$ A. J. Archer, D. Pini, R. Evans, and L. Reatto, J. Chem. Phys. 126, 014104 (2007).

${ }^{15}$ A. Ciach and W. T. Góźdź, Condens. Matter Phys. 13, 23603 (2010).

${ }^{16}$ J. Pȩkalski, A. Ciach, and N. G. Almarza, J. Chem. Phys. 138, 144903 (2013).

${ }^{17}$ J. Pȩkalski, A. Ciach, and N. G. Almarza, J. Chem. Phys. 140, 114701 (2014).

${ }^{18}$ N. G. Almarza, J. Pȩkalski, and A. Ciach, J. Chem. Phys. 140, 164708 (2014).

${ }^{19}$ J. Pȩkalski, A. Ciach, and N. G. Almarza, J. Chem. Phys. 142, 014903 (2015).

${ }^{20}$ D. de las Heras and M. Schmidt, Phys. Rev. Lett. 113, 238304 (2014).

${ }^{21}$ I. Williams, E. C. Oğuz, P. Bartlett, H. Löwen, and C. P. Royall, Nat. Commun. 4, 2555 (2013).

${ }^{22}$ A. Shukla, E. Mylonas, E. D. Cola, S. Finet, P. Timmins, T. Narayanan, and D. I. Svergun, Proc. Natl. Acad. Sci. U. S. A. 105, 5075 (2008).

${ }^{23}$ N. Metropolis, A. W. Rosenbluth, M. N. Rosenbluth, A. H. Teller, and E. Teller, J. Chem. Phys. 21, 1087 (1953).

${ }^{24}$ A. Gonzalez, J. A. White, F. L. Roman, and R. Evans, J. Chem. Phys. 109, 3637 (1998).

${ }^{25}$ Y. W. Kim, S. C. Kim, and S. H. Suh, J. Chem. Phys. 110, 1230 (1999).

${ }^{26} \mathrm{M}$. Seul and D. Andelman, Science 267, 476 (1995).

${ }^{27}$ A. Ciach, J. Pȩkalski, and W. T. Góźdź, Soft Matter 9, 6301 (2013).

${ }^{28}$ J. Pȩkalski, P. Rogowski, and A. Ciach, Mol. Phys. 113, 1022 (2014).

${ }^{29}$ S. A. Cannas, M. F. Michelon, D. A. Stariolo, and F. A. Tamarit, Phys. Rev. B 73, 184425 (2006).

${ }^{30}$ S. A. Cannas, M. Carubelli, O. V. Billoni, and D. A. Stariolo, Phys. Rev. B 84, 014404 (2011).

${ }^{31}$ D. G. Barci and D. A. Stariolo, Phys. Rev. Lett. 98, 200604 (2007).

${ }^{32}$ D. G. Barci and D. A. Stariolo, Phys. Rev. B 79, 075437 (2009). 